\title{
Observation of a new type of low-frequency waves at comet 67P/Churyumov-Gerasimenko
}

\author{
I. Richter ${ }^{1}$, C. Koenders ${ }^{1}$, H.-U. Auster ${ }^{1}$, D. Frühauff ${ }^{1}$, C. Götz ${ }^{1}$, P. Heinisch ${ }^{1}$, C. Perschke ${ }^{1,2}$, U. Motschmann ${ }^{2,3}$,

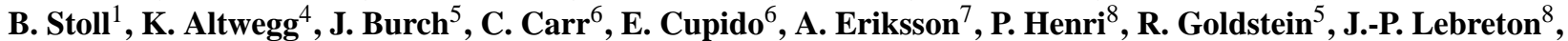 \\ P. Mokashi ${ }^{5}$, Z. Nemeth ${ }^{9}$, H. Nilsson ${ }^{10}$, M. Rubin ${ }^{4}$, K. Szegö ${ }^{9}$, B. T. Tsurutani ${ }^{11}$, C. Vallat $^{12}$, M. Volwerk ${ }^{13}$, and \\ K.-H. Glassmeier ${ }^{1}$ \\ ${ }^{1}$ Institut für Geophysik und extraterrestrische Physik, TU Braunschweig, Mendelssohnstr. 3, \\ 38106 Braunschweig, Germany \\ ${ }^{2}$ Institut für Theoretische Physik, TU Braunschweig, Mendelssohnstr. 3, 38106 Braunschweig, Germany \\ ${ }^{3}$ German Aerospace Center (DLR), Institute of Planetary Research, Rutherfordstraße 2, \\ 12489 Berlin, Germany \\ ${ }^{4}$ Physikalisches Institut, University of Bern, Sidlerstrasse 5, 3012 Bern, Switzerland \\ ${ }^{5}$ Southwest Research Institute, P.O. Drawer 28510, San Antonio, TX 78228-0510, USA \\ ${ }^{6}$ Imperial College London, Exhibition Road, London SW7 2AZ, UK \\ ${ }^{7}$ Swedish Institute of Space Physics, Ångström Laboratory, Lägerhyddsvägen 1, Uppsala, Sweden \\ ${ }^{8}$ Laboratoire de Physique et Chimie de l'Environnement et de l'Espace, UMR 7328 CNRS - \\ Université d'Orléans, Orléans, France \\ ${ }^{9}$ Wigner Research Centre for Physics, 1121 Konkoly Thege Street 29-33, Budapest, Hungary \\ ${ }^{10}$ Swedish Institute of Space Physics, P.O. Box 812, 98128 Kiruna, Sweden \\ ${ }^{11}$ Jet Propulsion Laboratory, California Institute of Technology, 4800 Oak Grove Drive, Pasadena, \\ CA 91109, USA \\ ${ }^{12}$ Rosetta Science Ground Segment, European Space Astronomy Centre, 28691 Villanueva de la Cañada, \\ Madrid, Spain \\ ${ }^{13}$ Space Research Institute, Austrian Academy of Sciences, Schmiedlstraße 6, 8042 Graz, Austria
}

Correspondence to: I. Richter (i.richter@tu-braunschweig.de)

Received: 22 May 2015 - Revised: 14 July 2015 - Accepted: 17 July 2015 - Published: 19 August 2015

\begin{abstract}
We report on magnetic field measurements made in the innermost coma of 67P/Churyumov-Gerasimenko in its low-activity state. Quasi-coherent, large-amplitude $(\delta B / B \sim 1)$, compressional magnetic field oscillations at $\sim 40 \mathrm{mHz}$ dominate the immediate plasma environment of the nucleus. This differs from previously studied cometary interaction regions where waves at the cometary ion gyrofrequencies are the main feature. Thus classical pickup-iondriven instabilities are unable to explain the observations. We propose a cross-field current instability associated with newborn cometary ion currents as a possible source mechanism.
\end{abstract}

Keywords. Magnetospheric physics (plasma waves and instabilities; solar wind interactions with unmagnetized bod- ies) - solar physics astrophysics and astronomy (magnetic fields)

\section{Introduction}

Typically ionization of atoms and molecules of cometary origin is the most important process for the interaction of strongly outgassing comets and the solar wind. During encounters of the ICE, Sakigake, and Giotto spacecraft with active comets 21P/Giacobini-Zinner, 1P/Halley, and 26P/Grigg-Skjellerup, large-amplitude plasma waves and turbulence have been one of the most pronounced obser- 
vational findings in the cometary magnetosphere (Tsurutani and Smith, 1986; Yumoto et al., 1986; Neubauer et al., 1986; Glassmeier et al., 1989; Glassmeier and Neubauer, 1993; Volwerk et al., 2014). Ion ring-beam instabilities (Wu and Davidson, 1972) and non-gyrotropic phase space densitydriven instabilities (Motschmann and Glassmeier, 1993) are the source mechanism of these waves. In the spacecraft $(\mathrm{s} / \mathrm{c})$ frame of reference (and pickup ion frame), those waves were detected at the cometary $\mathrm{H}_{2} \mathrm{O}^{+}$ion gyro-frequency.

Rosetta's journey (Glassmeier et al., 2007a) alongside comet 67P/Churyumov-Gerasimenko now allows for electromagnetic waves to be studied at the beginning of cometary activity, at the birth of the cometary magnetosphere (Nilsson et al., 2015). It should be noted that, under these low-activity conditions, typical solar wind-cometary interaction regions like the bow shock and magnetic pileup region are not expected (e.g., Koenders et al., 2013; Rubin et al., 2014) and also not observed (Nilsson et al., 2015). We shall report on wave observations at distances of 2.7-3.6 AU from the Sun and $10-1000 \mathrm{~km}$ from the comet.

\section{Mission and instrumentation}

Rosetta arrived at 67P/Churyumov-Gerasimenko on $6 \mathrm{Au}-$ gust 2014 at a heliocentric distance of 3.6 AU. The spacecraft was initially put into a $\sim 100 \mathrm{~km}$ orbit around the comet's nucleus. Observations reported here are limited to the dayside inner coma and sampled over a time span of a $\sim 4$ months (from August to November 2014). Using measurements of the Rosetta Orbiter Spectrometer for Ion and Neutral Analysis Cometary Pressure Sensor (ROSINA COPS) (Balsiger et al., 2007), the cometary activity at this heliocentric distance was determined to be below $4 \times 10^{26} \mathrm{~s}^{-1}$. This production rate is $2-3$ orders of magnitude lower than at any other previous cometary encounter where pickup ion waves were detected $\left(8 \times 10^{29} \mathrm{~s}^{-1}\right.$ at $1 \mathrm{P} /$ Halley, down to $7 \times 10^{27} \mathrm{~s}^{-1}$ at 26P/Grigg-Skjellerup) (see Richter et al., 2011).

The Rosetta orbiter is equipped with a suite of plasma instruments - the Rosetta Plasma Consortium (RPC) set of particle and field sensors (Carr et al., 2007). RPC-MAG, the tri-axial fluxgate magnetometer system (Glassmeier et al., 2007b), consists of two sensors mounted on a $1.5 \mathrm{~m}$ boom, separated by $0.15 \mathrm{~m}$. The short boom length implies that the spacecraft is heavily contaminating the magnetic field measurements. At this stage of the investigation it was not possible to completely remove these quasi-static spacecraft bias fields from the measured magnetic field values. The dynamic range of RPC-MAG is $\pm 16000 \mathrm{nT}$, and its resolution $0.03 \mathrm{nT}$. Although the magnetometer is capable of acquiring the magnetic field measurements with sampling rates up to $20 \mathrm{~Hz}$, the data presented here correspond to the instrument's normal operational mode, i.e., a sampling rate of $1 \mathrm{~Hz}$, which is sufficient for the purposes of our investigation. The magnetic field observations are represented in a comet-centered solar equatorial (CSEQ) coordinate system. The $+x$ axis points from the comet to the Sun, the $+z$ axis is the component of the Sun's north pole of date orthogonal to the $+x$ axis, and the $+y$ axis completes the right-handed reference frame. The origin of the coordinate system is the comet's center of mass.

The Rosetta Ion and Electron Sensor (RPC-IES) (Burch et al., 2006) and the Ion Composition Analyser (RPCICA) (Nilsson et al., 2006) provide information on cometary ions produced in the coma of 67P/Churyumov-Gerasimenko. Observations of the neutral gas number density made by ROSINA COPS are used as well.

\section{Observations}

Upon arrival at the comet on 6 August 2014, RPC-MAG started to detect large-amplitude, quasi-coherent magnetic field fluctuations. During the aforementioned observational period, we were able to collect $\sim 3000$ cases of wave activity with typical frequencies of $\sim 40 \mathrm{mHz}$. Figure 1 shows an example of these waves. The wave activity is clearly visible in all three components. Peak-to-peak amplitudes are of the order of $4 \mathrm{nT}$, which is about twice as large as the ambient solar wind magnetic field at this heliocentric distance. The oscillations are neither purely transverse nor purely compressional.

A preliminary minimum variance analysis between $\mathrm{Au}-$ gust and November 2014 does not show any preferred direction of wave propagation, with respect to neither the solar wind flow nor the mean magnetic field direction. A full discussion of the minimum variance analysis results will be presented elsewhere. As only single point observations are available, because only the ROSETTA orbiter magnetometer was operating and the lander was still attached to the orbiter, no information about a typical wavelength can be inferred at this time.

The quasi-coherent nature of these fluctuations is also clearly visible in power spectral density distributions (Fig. 2). The steep spectral slope at frequencies beyond the peak frequency is not uncommon for the observations at 67P/Churyumov-Gerasimenko; typical spectral slopes are between -3 and -5 . The wave spectra typically exhibit a single-peak structure as shown in Fig. 2.

The peak frequencies exhibit a Rayleigh-type distribution grouped around $40 \mathrm{mHz}$ (Fig. 2). Further statistical analyses do not show any clear correlation of the peak frequencies with the ambient magnetic field magnitude. Such a correlation would be expected if the observed frequency coincides with the local proton gyro-frequency $f_{\mathrm{p}}\left(f_{\mathrm{p}} \sim 40 \mathrm{mHz}\right.$ with $B=2.5 \mathrm{nT}$ ). Though the agreement between the peak frequency of the Rayleigh-type distribution and the local gyrofrequency is striking, we argue here that the observed waves are not in proton cyclotron resonance as a clear correlation between magnetic field magnitude and frequency is missing. 


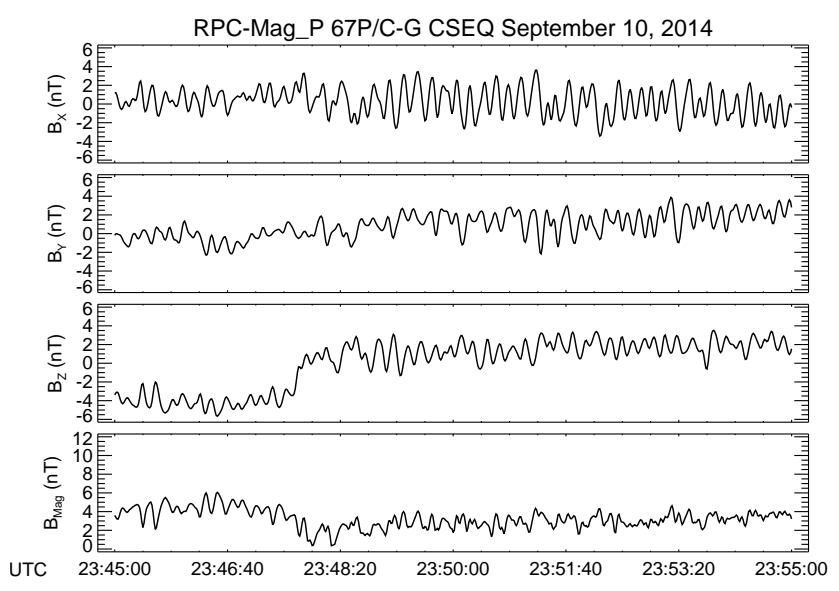

Figure 1. Example of magnetic field observations made onboard the Rosetta spacecraft on 10 September 2014, 23:45-23:55 UTC. The position vector of the spacecraft in the comet-centered solar equatorial (CSEQ; for details see text) coordinate system was $(3.9,-20.6,20.4) \mathrm{km}$.

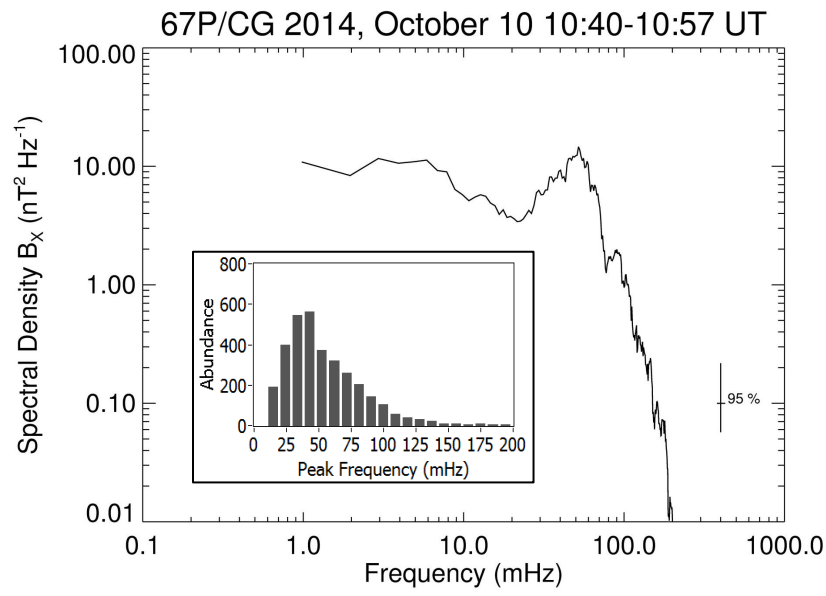

Figure 2. Example of a spectrum of magnetic field fluctuations observed in the innermost coma of 67P/Churyumov-Gerasimenko on 10 October 2014, 10:40-10:57 UTC (CSEQ position vector $(-0.3,-10.1,-1.7) \mathrm{km})$. The power spectrum has been calculated by application of a standard fast Fourier transform routine to a 17 min time interval. A box car window is used to average in the frequency domain. Eighteen degrees of freedom are used. The inset shows the distribution of peak spectral frequencies in the period August-November 2014. The confidence interval is shown in the bottom right corner.

Wave activity was first observed by the RPC-MAG instrument at a dayside distance of approximately $100 \mathrm{~km}$ from the nucleus and steadily increased up to a distance of $30 \mathrm{~km}$ (Fig. 3). Hourly trace spectral densities have been integrated over the frequency range $30-80 \mathrm{mHz}$ and divided by twice the vacuum permeability to determine the magnetic energy density of the observed fluctuations. Closer to the nucleus the magnetic energy density seems to saturate. The spread of the distribution at around 20 and $30 \mathrm{~km}$ is caused by Rosetta's trajectory with respect to the comet. During the first months after arriving at the comet the spacecraft was often been positioned in the so-called bound orbits with a fixed distance to the nucleus, mostly in the terminator plane (Hässig et al., 2015). Hence, more observations are made at these distances. The wave activity variations at constant distance are caused by temporal variations in the neutral gas density produced by localized gas sources on the nucleus' surface and modulated by the comet's rotation (Hässig et al., 2015), leading through the ionization of cometary neutrals to temporal variations in the cometary plasma density. The variations also reflect a dependence on solar wind variations as well as elevation and azimuth angle of Rosetta's position. Future work is planned to separate these dependencies. For the range of $30-100 \mathrm{~km}$ the spatial variation in the magnetic energy density is found to be $W \propto r^{-\alpha}$ with $\alpha=8.04 \pm 0.27$ (for a $95 \%$ confidence interval $\alpha \in[7.77 ; 8.31]$, yielding a relative error of $100 \cdot \frac{\delta \alpha}{\alpha}=3.3 \%$ ), equivalent to a quartic decrease in the wave amplitude with distance.

Comparing wave intensity with the neutral gas number density as measured by ROSINA COPS exhibits a clear global relation between both quantities. COPS also detected a neutral gas number density, $N$, above its noise level at a distance of about $100 \mathrm{~km}$ (Fig. 3). The neutral density decreases as $N \propto r^{-\beta}$ with $\beta=1.43 \pm 0.07$ (for a $95 \%$ confidence interval $\beta \in[1.36 ; 1.50])$. The deviation 100 . $\frac{\beta_{\text {theo }}-\beta_{\text {calc }}}{\beta_{\text {theo }}}=28.5 \%$ from the theoretically expected value $\beta_{\text {theo }}=2$ (Coates and Jones, 2009) is due to Rosetta first approaching from the afternoon side ( $45^{\circ}$ phase angle) before moving to the terminator plane $\left(90^{\circ}\right.$ phase angle) and therefore encountering different insolation conditions at the sub-spacecraft location.

Here only a global relation is discussed. More detailed analyses on the relation between neutral gas density and magnetic field variations are currently being prepared; however, such studies require consideration of the rotation of the nucleus with a period of about $12.4 \mathrm{~h}$ as well as any inhomogeneity in the active regions.

\section{Discussion and possible wave source mechanism}

Comparing the radial variations in both the magnetic energy density, $W$, and the neutral gas density suggests an approximate global relation $W \propto N^{-\gamma}$ with $\gamma=5.62 \pm 0.45$ (for a $95 \%$ confidence interval $\gamma \in[5.17 ; 6.07])$. A more detailed correlation analysis between the individual values of both quantities at the same radial distance reveals a linear Pearson correlation coefficient of $r=0.52$. This correlation reflects the large spread of both wave energy and neutral gas density. As waves are generated locally under time-varying solar wind conditions and propagate within the inner coma, this weak correlation is not surprising. Because the production rate of heavy cometary ions is proportional to the neutral 


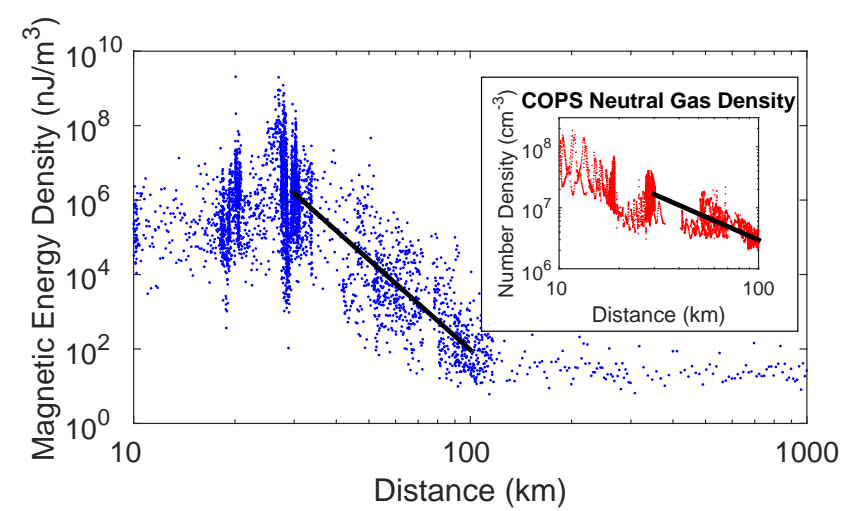

Figure 3. Radial variation in the magnetic energy density of the low-frequency wave activity in the spectral range $30-80 \mathrm{mHz}$. The inset shows the radial variation in the density of cometary neutrals as measured by the neutral gas monitor COPS of the ROSINA instrument.

gas density, we conclude that wave activity in general is controlled by the cometary ion production rate.

The observed frequencies concentrate between 25 and $75 \mathrm{mHz}$ (Fig. 2). In the coma the local $\mathrm{H}_{2} \mathrm{O}^{+}$ion gyrofrequency is in the range of $0.8-3.2 \mathrm{mHz}$. Thus, there are clear differences between the observed frequencies at the birth stage of the cometary magnetosphere and the heavy ion cyclotron frequencies, as expected for well-developed cometary interaction regions. This points towards a new generation mechanism for the type of wave reported here for a weakly outgassing comet.

The size of the innermost interaction region is much less than the Larmor radius of the newborn ions. The pickup ion's initial velocity is about $0.8 \mathrm{~km} \mathrm{~s}^{-1}$; acceleration by the interplanetary electric field, $\boldsymbol{E}$, up to velocities, $|\boldsymbol{v}|$, of a few tens of kilometers per second is observed (Nilsson et al., 2015) by RPC-IES and RPC-ICA. After ionization the newborn ions are moving transverse to the ambient magnetic field, $\boldsymbol{B}$, and the solar wind flow in the direction of the electric field, constituting a cross-field electric current density. The cometary ion motion is controlled by the electric field. Lorentz forces are not yet important in this innermost coma region. The ratio of the electric force to the Lorentz force is of the order of the ratio of the gyro-period $T_{\mathrm{G}}$ to the lifetime $\tau$ of the newborn ions: $|\boldsymbol{E}| /|\boldsymbol{v} \times \boldsymbol{B}| \approx T_{\mathrm{G}} / \tau$. Newborn cometary ions with $\tau \ll T_{\mathrm{G}}$ are essentially unmagnetized. Lorentz forces become important only on scales comparable to and larger than a cometary ion Larmor radius. Within distances to the nucleus smaller than a Larmor radius, i.e., in the Larmor sphere (Sauer et al., 1998), physical processes different from those generating the classical pickup-ion-related ion cyclotron waves are important.

A newborn water ion flux, $n \cdot v$, of at least $10^{10} \mathrm{~m}^{-2} \mathrm{~s}^{-1}$ has been observed by the RPC-ICA sensor (Nilsson et al., 2015). Due to the sensor's limited field of view, the actual flux will be higher. Assuming a flux density $3 \times 10^{10} \mathrm{~m}^{-2} \mathrm{~s}^{-1}$ and singly charged ions the electric current density is estimated to be about $j \sim 4.8 \times 10^{-9} \mathrm{~A} \mathrm{~m}^{-2}$. Electromagnetic instabilities attributable to such a cross-field current have been studied in the past (Chang et al., 1990; Sauer et al., 1998), but not for conditions typical for the plasma situation at $67 \mathrm{P} /$ Churyumov-Gerasimenko. Assuming that the crossfield current is driven unstable, a transverse wave number $k_{\perp}$ can be estimated using Ampeère's law: $k_{\perp}=\mu_{0} \delta j / \delta B$. With $\delta j \sim j$ and $\delta B \approx 1 \mathrm{nT}$, a value of $k_{\perp} \approx 6 \times 10^{-6} \mathrm{~m}^{-1}$ results, corresponding to a transverse wave length of $524 \mathrm{~km}$. It should be noted that this wave number component is transverse to the mean magnetic field as well as the cross-field current. The polarization of the observed waves is neither purely compressional nor purely transverse. This points towards offangle propagation. We therefore assume that all three components of the wave vector are of comparable magnitude.

The wavelength reported here is larger than the scale of the dayside inner coma where wave activity has been observed. However, the generation region is probably significantly larger. But as waves generated trough cometary interaction at larger distances are buried in the pre-existing solar wind turbulence, the signal was not detected by RPC-MAG. We need to await a further increase in the cometary activity to see a further expansion of that region where cometary waves clearly stand out from the solar wind turbulence background.

The frequency of the unstable mode may be estimated using the beam-mode dispersion relation (Chang et al., 1990; Sauer et al., 1998) $\omega \approx k_{\|} \cdot v$, where $k_{\|}$is the beam parallel wave vector component and $v$ the cometary ion beam velocity.

Using an observational estimate (Nilsson et al., 2015) of the ion velocity $v=40 \mathrm{~km} \mathrm{~s}^{-1}$, we obtain an angular frequency omega $\omega \sim 0.24 \operatorname{rads}^{-1}$, i.e., a wave frequency $f \sim$ $38 \mathrm{mHz}$.

This value is comparable to the observed frequencies.

It should be noted that the suggested wave source is not co-moving with the solar wind flow. The cross-field current source is due to freshly ionized cometary ions which are not yet moving with the solar wind velocity as they did not have time to be accelerated to solar wind speed on the time and distance scales we are looking at. The wave source is almost fixed in the nucleus frame of reference. Therefore, Doppler effects can be neglected as the Rosetta spacecraft is only slowly moving with respect to the nucleus $\left(v_{\mathrm{s} / \mathrm{c}} \sim 1 \mathrm{~m} \mathrm{~s}^{-1}\right)$.

As the wave number is proportional to the current density perturbation, $k \propto \delta j \propto n v$, the dispersion relation provides the following approximate expression between wave frequency, ion density and ion velocity: $f \propto n v^{2}$. Assuming a constant electric field accelerating the particles, the ion velocity should increase linearly with distance. For an ion density decreasing with the square of the distance, the frequencies should therefore not exhibit any major dependence on distance, which is what we observe. Of course, this is only a first conjecture stimulating future analysis. 
Our model furthermore allows for the apparent saturation of wave activity at around $30 \mathrm{~km}$ distance to be understood: the newborn ions need to be accelerated to constitute a significant current and the waves need to grow. However, further detailed theoretical modeling is required to validate the conjecture of a cross-field current-driven instability causing the newly detected low-frequency wave activity in the Larmor sphere of 67P/Churyumov-Gerasimenko.

Acknowledgements. The RPC-MAG and ROSINA data will be made available through the PSA archive of ESA and the PDS archive of NASA. Rosetta is a European Space Agency (ESA) mission with contributions from its member states and the National Aeronautics and Space Administration (NASA). The work on RPCMAG was financially supported by the German Ministerium für Wirtschaft und Energie and the Deutsches Zentrum für Luft- und Raumfahrt under contract 50QP 1401. The work on ROSINA was funded by the federal state of Bern, the Swiss National Science Foundation, and the ESA PRODEX program. Portions of this research were performed at the Jet Propulsion Laboratory, California Institute of Technology, under contract with NASA. We are indebted to the whole of the Rosetta Mission Team, SGS, and RMOC for their outstanding efforts in making this mission possible.

The topical editor E. Roussos thanks the two anonymous referees for help in evaluating this paper.

\section{References}

Balsiger, H., Altwegg, K., Bochsler, P., Eberhardt, P., Fischer, J., Graf, S., Jäckel, A., Kopp, E., Langer, U., Mildner, M., Müller, J., Riesen, T., Rubin, M., Scherer, S., Wurz, P., Wüthrich, S., Arijs, E., Delanoye, S., Keyser, J. D., Neefs, E., Nevejans, D., Rème, H., Aoustin, C., Mazelle, C., Médale, J.-L., Sauvaud, J. A., Berthelier, J.-J., Bertaux, J.-L., Duvet, L., Illiano, J.-M., Fuselier, S. A., Ghielmetti, A. G., Magoncelli, T., Shelley, E. G., Korth, A., Heerlein, K., Lauche, H., Livi, S., Loose, A., Mall, U., Wilken, B., Gliem, F., Fiethe, B., Gombosi, T. I., Block, B., Carignan, G. R., Fisk, L. A., Waite, J. H., Young, D. T., and Wollnik, H.: Rosina - Rosetta Orbiter Spectrometer for Ion and Neutral Analysis, Space Sci. Rev., 128, 745801, doi:10.1007/s11214-006-8335-3, 2007.

Burch, J. L., Goldstein, R., Cravens, T. E., Gibson, W. C., Lundin, R. N., Pollock, C. J., Winningham, J. D., and Young, D. T.: RPCIES: The Ion and Electron Sensor of the Rosetta Plasma Consortium, Space Sci. Rev., 128, 697-712, doi:10.1007/s11214-0069002-4, 2006.

Carr, C., Cupido, E., Lee, C. G. Y., Balogh, A., Beek, T., Burch, J. L., Dunford, C. N., Eriksson, A. I., Gill, R., Glassmeier, K.-H., Goldstein, R., Lagoutte, D., Lundin, R., Lundin, K., Lybekk, B., Michau, J. L., Musmann, G., Nilsson, H., Pollock, C., Richter, I., and Trotignon, J. G.: RPC: The Rosetta Plasma Consortium, Space Sci. Rev., 128, 629-647, doi:10.1007/s11214-006-9136-4, 2007.

Chang, C., Wong, H., and Wu, C.: Electromagnetic Instabilities Attributed to a Cross-Field Ion Drift, Phys. Rev. Lett., 65, 11041107, doi:10.1103/PhysRevLett.65.1104, 1990.
Coates, A. J. and Jones, G. H.: Plasma environment of Jupiter family comets, Planet. Space Sci., 57, 1175-1191, doi:10.1016/j.pss.2009.04.009, 2009.

Glassmeier, K.-H. and Neubauer, F. M.: Low-Frequency Electromagnetic Plasma Waves at Comet P/Grigg-Skjellerup: Overview and Spectral Characteristics, J. Geophys. Res., 98, 20921-20935, doi:10.1029/93JA02583, 1993.

Glassmeier, K.-H., Coates, A. J., Acuña, M. H., Goldstein, M. L., Johnstone, A. D., Neubauer, F. M., and Rème, H.: Spectral characteristics of low-frequency plasma turbulence upstream of comet P/Halley, J. Geophys. Res., 94, 37, doi:10.1029/JA094iA01p00037, 1989.

Glassmeier, K.-H., Boehnhardt, H., Koschny, D., Kührt, E., and Richter, I.: The Rosetta Mission: Flying Towards the Origin of the Solar System, Space Sci. Rev., 128, 1-21, doi:10.1007/s11214-006-9140-8, 2007a.

Glassmeier, K.-H., Richter, I., Diedrich, A., Musmann, G., Auster, U., Motschmann, U., Balogh, A., Carr, C., Cupido, E., Coates, A., Rother, M., Schwingenschuh, K., Szegö, K., and Tsurutani, B. T.: RPC-MAG The Fluxgate Magnetometer in the ROSETTA Plasma Consortium, Space Sci. Rev., 128, 649-670, doi:10.1007/s11214-006-9114-x, 2007b.

Hässig, M., Altwegg, K., Balsiger, H., Bar-Nun, A., Berthelier, J. J., Bieler, A., Bochsler, P., Briois, C., Calmonte, U., Combi, M., De Keyser, J., Eberhardt, P., Fiethe, B., Fuselier, S. A., Galand, M., Gasc, S., Gombosi, T. I., Hansen, K. C., Jäckel, A., Keller, H. U., Kopp, E., Korth, A., Kührt, E., Le Roy, L., Mall, U., Marty, B., Mousis, O., Neefs, E., Owen, T., Rème, H., Rubin, M., Sémon, T., Tornow, C., Tzou, C.-Y., Waite, J. H., and Wurz, P.: Cometary science. Time variability and heterogeneity in the coma of 67P/Churyumov-Gerasimenko, Science, 347, aaa0276, doi:10.1126/science.aaa0276, 2015.

Koenders, C., Glassmeier, K.-H., Richter, I., Motschmann, U., and Rubin, M.: Revisiting cometary bow shock positions, Planet. Space Sci., 87, 85-95, doi:10.1016/j.pss.2013.08.009, 2013.

Motschmann, U. and Glassmeier, K.-H.: Nongyrotropic Distribution of Pickup Ions at Comet P/Grigg-Skjellerup: A Possible Source of Wave Activity, J. Geophys. Res., 98, 20977-20983, doi:10.1029/93JA02533, 1993.

Neubauer, F. M., Glassmeier, K.-H., Pohl, M., Raeder, J., Acuna, M. H., Burlaga, L. F., Ness, N. F., Musmann, G., Mariani, F., Wallis, M. K., Ungstrup, E., and Schmidt, H. U.: First results from the Giotto magnetometer experiment at comet Halley, Nature, 321, 352-355, doi:10.1038/321352a0, 1986.

Nilsson, H., Lundin, R., Lundin, K., Barabash, S., Borg, H., Norberg, O., Fedorov, A., Sauvaud, J.-A., Koskinen, H., Kallio, E., Riihelä, P., and Burch, J. L.: RPC-ICA: The Ion Composition Analyzer of the Rosetta Plasma Consortium, Space Sci. Rev., 128, 671-695, doi:10.1007/s11214-006-9031-z, 2006.

Nilsson, H., Stenberg Wieser, G., Behar, E., Wedlund, C. S., Gunell, H., Yamauchi, M., Lundin, R., Barabash, S., Wieser, M., Carr, C., Cupido, E., Burch, J. L., Fedorov, A., Sauvaud, J.-A., Koskinen, H., Kallio, E., Lebreton, J.-P., Eriksson, A., Edberg, N., Goldstein, R., Henri, P., Koenders, C., Mokashi, P., Nemeth, Z., Richter, I., Szego, K., Volwerk, M., Vallat, C., and Rubin, M.: Birth of a comet magnetosphere: A spring of water ions, Science, 347, doi:10.1126/science.aaa0571, 2015.

Richter, I., Koenders, C., Glassmeier, K.-H., Tsurutani, B. T., and Goldstein, R.: Deep Space 1 at comet 19P/Borrelly: Magnetic 
field and plasma observations, Planet. Space Sci., 59, 691-698, doi:10.1016/j.pss.2011.02.001, 2011.

Rubin, M., Koenders, C., Altwegg, K., Combi, M., Glassmeier, K.-H., Gombosi, T., Hansen, K., Motschmann, U., Richter, I., Tenishev, V., and Tóth, G.: Plasma environment of a weak comet - Predictions for Comet 67P/Churyumov-Gerasimenko from multifluid-MHD and Hybrid models, Icarus, 242, 38-49, doi:10.1016/j.icarus.2014.07.021, 2014.

Sauer, K., Dubinin, E., Baumgärtel, K., and Tarasov, V.: Lowfrequency electromagnetic waves and instabilities within the Martian bi-ion plasma, Earth, Planets and Space, 50, 269-278, doi:10.1186/BF03352113, 1998.

Tsurutani, B. T. and Smith, E. J.: Strong hydromagnetic turbulence associated with comet Giacobini-Zinner, Geophys. Res. Lett., 13, 259-262, doi:10.1029/GL013i003p00259, 1986.
Volwerk, M., Glassmeier, K.-H., Delva, M., Schmid, D., Koenders, C., Richter, I., and Szegö, K.: A comparison between VEGA 1, 2 and Giotto flybys of comet 1P/Halley: implications for Rosetta, Ann. Geophys., 32, 1441-1453, doi:10.5194/angeo-321441-2014, 2014.

Wu, C. S. and Davidson, R. C.: Electromagnetic instabilities produced by neutral-particle ionization in interplanetary space, J. Geophys. Res., 77, 5399-5406, doi:10.1029/JA077i028p05399, 1972.

Yumoto, K., Saito, T., and Nakagawa, T.: Hydromagnetic waves near $\mathrm{O}^{+}\left(\mathrm{OR} \mathrm{H}_{2} \mathrm{O}^{+}\right)$ion cyclotron frequency observed by Sakigake at the closest approach to comet Halley, Geophys. Res. Lett., 13, 825-828, doi:10.1029/GL013i008p00825, 1986. 